MUSICA THEORICA 2021, V. 6.1

SCIENTIFIC ARTICLE

DOI: $10.52930 / \mathrm{mt} . v 6 \mathrm{i} 1.175$

Data do recebimento: 18/07/2021

Data da aprovação final: 18/09/2021

\title{
Composição algorítmica de progressões harmônicas ao estilo de Antonio Carlos Jobim através de processos markovianos
}

\author{
Algorithmic Composition of Harmonic Progressions through Markovian \\ Processes in the Style of Antonio Carlos Jobim
}

\author{
Ana Miccolis \\ Claudia Usai \\ Eduardo Cabral \\ Igor Chagas \\ João Penchel \\ Max Kühn \\ Vinicius Ramos Braga \\ Carlos Almada \\ Universidade Federal do Rio de Janeiro
}

\begin{abstract}
Resumo: O presente artigo é vinculado a uma pesquisa recentemente concluída, destinada à análise da obra de Antonio Carlos Jobim (1927-1994) focando nas relações harmônicas. O desenvolvimento metodológico da pesquisa foi efetivado em dois estágios: análise assistida por computador e coleta estatística dos dados analisados. O processo analítico foi estabelecido em um quadro teórico-metodológico (denominado Sistema J), cujas bases são descritas em detalhes em trabalho recente (Almada et al. 2019a). Princípios, procedimentos e conceitos originais, entre os quais qualidade-classe e relação binária, embasaram a produção de dados. O principal objetivo deste trabalho é apresentar uma das aplicações composicionais advindas do processamento e discussão dos dados obtidos ao longo do mapeamento estatístico. Trata-se de JobKov, um software destinado à composição algorítmica de progressões acordais originais estilisticamente jobinianas por intermédio de processos estocásticos markovianos. O artigo apresenta e descreve os algoritmos criados e a estrutura do programa, discutindo ainda como fatores estatísticos são cruciais para a determinação de estilos composicionais.
\end{abstract}

Palavras-chave: Antonio Carlos Jobim. Análises harmônica e estatística assistidas por computador. Qualidade-classe. Relação binária. Processos markovianos. 
Abstract: This article is associated with a recently concluded analysis of the music by Antonio Carlos Jobim (1927-1994) focusing on harmonic relationships. The methodological development of the research was accomplished in two stages: computer-assisted analysis and statistical survey of the analyzed data. The analytical process was grounded on a theoretical-methodological framework (called System J), whose basis are described in detail in a recent publication (Almada et al. 2019a). Principles, procedures, and concepts, amongst them the notions of quality-class and binary relation, oriented the data production. The main objective of this article is to present one of the compositional applications arising from the processing and discussion of the data obtained along the statistical mapping. This is the software JobKov, designed for algorithmic composition of stylistically Jobinian original chord progressions through Markovian stochastic processes. The article introduces and describes the algorithms created and the structure of the program, also discussing how statistical factors are of paramount importance for determining compositional styles.

Keywords: Antonio Carlos Jobim. Computer-assisted harmonic and statistical analyses. Quality-classes. Binary relation. Markov processes.

\section{Introdução}

O presente artigo é associado a um projeto de pesquisa recentemente concluído, destinado ao exame analítico da integral das canções de Antonio Carlos Jobim (Jobim 2006) sob o ponto de vista das relações harmônicas. A pesquisa, teoricamente baseada em princípios transformacionais (Lewin 1987; Rinks 2011), teve como objetivos específicos a identificação e o mapeamento dos tipos de relações de transformação binária entre acordes contíguos, bem como classificar as estruturas internas dos próprios acordes. Essencialmente, o projeto se desenvolveu em duas etapas: análise assistida por computador e coleta estatística dos dados analisados, seguindo os moldes de trabalhos semelhantes, porém envolvendo diferentes repertórios (Temperley; Clercq 2013; Moss et al 2019). Para a realização do processo analítico foi elaborado um sistema teóricometodológico denominado Sistema J (S-J), que será descrito oportunamente. ${ }^{1}$

O universo harmônico das canções de Jobim serviu de modelo para a formação de um léxico de qualidades acordais empregadas pelo compositor. Foram catalogados 94 tipos de acordes (congregando básicos e variantes) encontrados em suas canções. Paralelamente, as relações binárias entre acordes contíguos foram devidamente mapeadas através de um software especialmente designado

\footnotetext{
${ }^{1}$ Para publicações sobre diversos aspectos teóricos e analíticos da pesquisa ver Almada et al. (2020; 2019a; 2019b), Almada (2017a; 2017b), Kühn et al. (2017a; 2017b) e Usai et al. (2020).
} 
para a tarefa de análise assistida, que fornece elementos estruturais de grande relevância para a caracterização e qualificação dos processos harmônicos jobinianos (Almada et al. 2019a).

A partir desses antecedentes, o presente artigo introduz uma das aplicações composicionais advindas do processamento e exame dos diversos dados obtidos ao longo do mapeamento estatístico efetuado. Trata-se de um novo software (denominado JobKov), destinado à produção de progressões acordais originais com qualquer extensão (em números de acordes) possível, e estilisticamente jobinianas, por assim dizer. JobKov opera por intermédio de um processo estocástico original, baseado em cadeias de Markov, que essencialmente visa a prever o estado imediatamente posterior a um estado atual, a partir do conhecimento estatístico de um determinado corpus de dados.

O principal objetivo deste artigo é justamente descrever em linhas gerais o Sistema J, bem como o processo composicional de progressões harmônicas originais que é operado por Jobkov. Em conjunto com os demais dados obtidos durante a pesquisa, o presente estudo busca contribuir para a sistematização dos estudos em música popular e, especificamente, para o entendimento do estilo harmônico de Jobim, por certo uma de suas características composicionais mais distintivas.

\section{O Cancioneiro Jobim}

A obra jobiniana apresenta uma variedade de gêneros e estilos. Sua formação contemplou aulas com professores de grande renome, como HansJoachim Koellreutter (teoria e notação musical), Paulo Silva (harmonia tradicional), Lucia Branco e Tomás Terán, ambos seus professores de piano. O conhecimento dos recursos de notação musical permitiu que Jobim pudesse registrar suas criações, assim como logo trabalhar transcrevendo para a partitura a música de compositores que não dominavam a escrita. Jobim foi fortemente influenciado tanto por grandes nomes da música popular brasileira (entre os quais, Ary Barroso, Dorival Caymmi e Noel Rosa), quanto por compositores da música de concerto. Desde o princípio dos estudos de piano, Jobim teve contato 
com obras de Bach, Beethoven, Chopin, Debussy, Ravel, Rachmaninoff, entre outros. ${ }^{2}$

Essa dupla fonte de influências contribuiu para que sua linguagem como compositor fosse construída no diálogo entre a tradição da música erudita e da prática da música popular, porém é possível observar que seu pensamento composicional está mais próximo da tradição escrita do que da tradição oral. Sendo um compositor que escrevia suas próprias partituras, Jobim deixou o projeto de lançamento de um cancioneiro, contendo todas as obras com arranjos fiéis às gravações originais, preservando os traços característicos de seu estilo. Essa publicação idealizada se tornaria, postumamente, o Cancioneiro Jobim: Obras Completas, publicado em 2006, sob direção de seu filho Paulo. O cancioneiro abrange cinco volumes que contêm toda a produção gravada do compositor.

Tal material consiste justamente na fonte referencial para as análises harmônicas empregadas na pesquisa. É importante destacar que adotamos a segmentação proposta no Cancioneiro, que organiza a obra jobiniana em cinco fases composicionais-temporais distintas, como se segue:

- FASE 1 (1947-1958): Engloba as peças da juventude, que contemplam gêneros como valsa, música de câmara e samba-canção. Grande parte das peças foi gravada por diversos cantores, mas podemos ressaltar os discos Sinfonia do Rio de Janeiro, com Billy Blanco (1954), Canção do Amor Demais, de Eliseth Cardoso (1958), Amor de Gente Moça, de Sylvia Telles (1959).

- FASE 2 (1959-1965): Compreende as peças produzidas durante o período da bossa nova (certamente, o que mais notabilizou Jobim), destacando as parcerias com Vinicius de Moraes e Newton Mendonça. Há os famosos álbuns de João Gilberto: Chega de Saudade (1959), O Amor O Sorriso e a Flor (1960), João Gilberto (1961), Getz/Gilberto (1963); bem como os autorais: Brasília Sinfonia da Alvorada (1961), The Composer of Desafinado Plays (1963), The Wonderful World of Antonio Carlos Jobim (1965), A Certain Mr. Jobim (1967).

- FASE 3 (1966-1970): Abrange as composições durante a estadia de Jobim em Nova York na metade da década de 1960. Podemos destacar o marcante disco dividido com Frank Sinatra (Francis Albert Sinatra $\mathcal{E}$ Antonio Carlos Jobim, de

\footnotetext{
2 Para maiores informações sobre aspectos biográficos, formativos e estéticos relacionados a Jobim, ver, entre outras fontes, Jobim 1996, Cabral 1997, Castro 1990; 2001; 2015.
} 
1967) e a experiência com a linguagem do cool jazz na trilogia produzida por Creed Taylor: Wave (1967), Stone Flower (1970) e Tide (1970).

- FASE 4 (1971-1983): Fase na qual o olhar do compositor parece se voltar para os ritmos brasileiros de matrizes folclóricas e nordestinas. É importante destacar a influência da linguagem de Heitor Villa-Lobos nas abordagens sinfônicas apresentadas nessa fase. O período conta com os álbuns Matita Perê (1973), Urubu (1976) e Terra Brasilis (1980).

- FASE 5 (1984-1994): Contempla as peças gravadas e compostas com a Banda Nova. É um período marcado pelo resgate de diversos gêneros utilizados nas fases anteriores, adicionados a outros inéditos, como o foxtrot. Algumas peças foram publicadas postumamente e não possuem gravações feitas pelo autor. Os discos que contemplam esse período são Passarim (1987) e Antonio Brasileiro (1994).

O Quadro 1 apresenta a lista completa das canções de acordo com a segmentação acima apresentada.

\section{Cadeias de Markov}

À primeira vista, a composição algorítmica ou, como no presente caso, a produção de progressões acordais originais em um estilo específico, podem parecer extremamente complexas. Afinal, como "ensinar" uma máquina a escolher entre as infinitas possibilidades de acordes e tensões para criar um todo musical de forma coesa? No entanto, eventos complexos podem ser simplificados por intermédio uma sistematização estocástica, no qual as leis da probabilidade possibilitam estabelecer regras estilísticas associadas a um determinado compositor. Uma vez que tal sistema seja alimentado com um corpus de obras relativamente grande, as escolhas composicionais podem ser mapeadas e analisadas através de regras matemáticas, que na prática, se tornam o próprio estilo de criação.

A utilização da estatística para uma compreensão mais profunda dos eventos harmônicos em uma obra já acontece desde os meados do século XX. Neste trabalho, optamos por utilizar as Cadeias de Markov para que o 
MUSICA THEORICA Revista da Associação Brasileira de Teoria e Análise Musical 2021, v. 6, n. 1, p. 218-238 - Journal of the Brazilian Society for Music

mapeamento do conjunto de estados (os acordes) e sua modelagem possam ser otimizados. ${ }^{3}$

\begin{tabular}{|c|c|c|c|c|}
\hline FASE 1 (1947-58) & Vida bela & Perdido nos teus olhos & Só tinha de ser com você & Two kites \\
\hline Imagina & Canção do amor demais & Samba de uma nota só & Bonita & Eu te amo \\
\hline Faz uma semana & Aula de matemática & Meditação & Samba do avião & Oficina \\
\hline Pensando em você & Cala meu amor & Corcovado & Inútil paisagem & Ai quem me dera \\
\hline Engano & Caminhos cruzados & Discussão & Por toda a minha vida & A cachorrinha \\
\hline Solidão & Mágoa & Incerteza & Esperança perdida & Espelho das águas \\
\hline Se é por falta de adeus & É preciso dizer adeus & Só em teus braços & Fotografia & Maria é dia \\
\hline O que vai ser de mim & Maria da Graça & Amor sem adeus & Estrada do sol & FASE 5 (1983-94) \\
\hline Lamento no morro & Chega de saudade & O grande amor & Por causa de você & Meninos eu vi \\
\hline Um nome de mulher & Outra vez & Isto eu não faço não & Retrato em branco e preto & A violeira \\
\hline Modinha & Tereza da praia & Na hora do adeus & FASE 3 (1966-70) & Cavaleiro monge \\
\hline Se todos fossem iguais a você & FASE 2 (1959-65) & Samba torto & Wave & O rio da minha aldeia \\
\hline Mulher sempre mulher & A chuva caiu & Este seu olhar & Olho pro céu & O tempo e o vento \\
\hline Eu e o meu amor & Desafinado & $\mathrm{O}$ amor em paz & Triste & Rodrigo, meu capitão \\
\hline Não devo sonhar & Brigas nunca mais & Insensatez & Chovendo na roseira & Um certo capitão Rodrigo \\
\hline Samba não é brinquedo & A felicidade & Acho que sim & Olha Maria & Senhora dona Bibiana \\
\hline Foi a noite & O nosso amor & Domingo azul do mar & Sabiá & Passarim \\
\hline Sonho desfeito & Frevo & Derradeira primavera & Pois é & Bebel \\
\hline Só saudade & Canta, canta mais & Andam dizendo & FASE 4 (1971-82) & Borzeguim \\
\hline Teu castigo & Cai a tarde & Canção em modo menor & Águas de março & Anos dourados \\
\hline Frase perdida & Sem você & Valsa do amor de nós dois & Ana Luiza & Chansong \\
\hline Vem viver ao meu lado & Soneto de separação & Velho riacho & Matita Perê & Luiza \\
\hline Luar e batucada & Demais & Pra mode chatear & Chora coração & Gabriela \\
\hline Eu não existo sem você & De você eu gosto & Vivo sonhando & Boto & Absolute Lee \\
\hline Sucedeu assim & Esquecendo você & Garota de Ipanema & Ligia & Piano na Mangueira \\
\hline As praias desertas & O que tinha de ser & Água de beber & A correnteza & Querida \\
\hline Caminho de pedra & Eu preciso de você & O morro não tem vez & Angela & Samba de Maria Luiza \\
\hline Luciana & Eu sei que vou te amar & Só danço samba & Dinheiro em penca & Forever green \\
\hline Janelas abertas & Canção da eterna despedida & Dindi & Você vai ver & Pato preto \\
\hline Estrada branca & Pelos caminhos da vida & Ela é carioca & Falando de amor & Trem de ferro \\
\hline
\end{tabular}

Quadro 1: Corpus das canções jobinianas analisadas.

Considere uma sequência de eventos aleatórios $\left\{X_{1}, X_{2}, X_{3}, \ldots\right\}$ que satisfaçam as seguintes condições:

1. Cada estado $x_{i}$ pertence a um espaço de estados $\left\{x_{1}, x_{2}, x_{3}, \ldots, x_{n}\right\}$, finito, onde diz-se que o sistema está no estado $\mathrm{q}_{\mathrm{i}}$ no $n$-ésimo passo, se $\mathrm{X}_{\mathrm{n}}=\mathrm{x}_{\mathrm{i}}$.

2. O estado que sucede o estado $n$, denotado por $X_{n+1}$ depende apenas do estado atual $\left(X_{n}\right)$ e, para cada par de estados $\left(x_{i}, X_{j}\right)$, existe uma probabilidade $\mathrm{p}_{\mathrm{i}}$ de que $x_{j}$ aconteça imediatamente após $x_{i}$. A Fig. 1 exemplifica esta condição considerando três estados possíveis $\left(\mathrm{x}_{1}, \mathrm{x}_{2}, \mathrm{x}_{3}\right)$ em dois pontos temporais contíguos, representados por $\mathrm{X}_{\mathrm{n}}$ e $\mathrm{X}_{\mathrm{n}+1}$.

3 O uso de processos markovianos para análise e composição musical é um campo consideravelmente vasto de pesquisa e aplicação, abrangendo diversos vieses e repertórios. Para um painel amplo sobre abordagens nesse sentido, ver Carvalho 2019. 


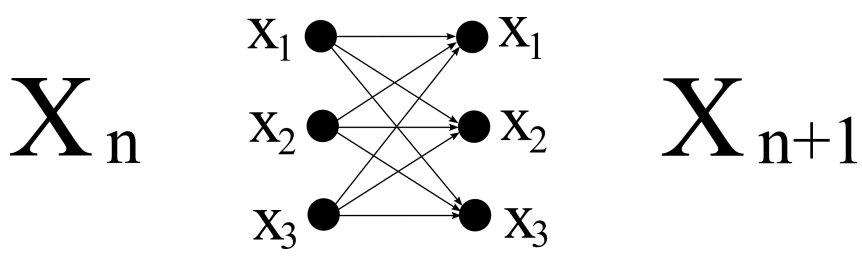

Figura 1: Exemplo de transições possíveis, considerando a existência de três únicos estados.

A Fig. 2 propõe uma representação alternativa do esquema da Fig. 1, como um grafo, incluindo as probabilidades de transição entre os estados.

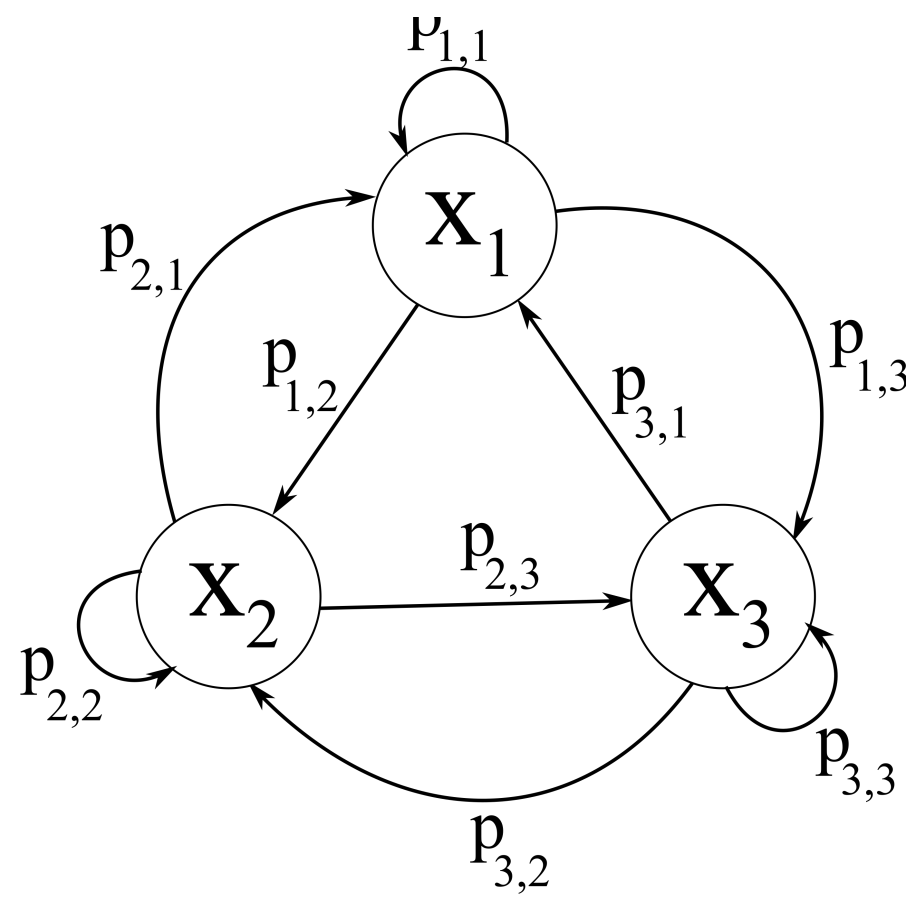

Figura 2: Reformulação do exemplo da Fig. 1 como um grafo, incluindo indicações de probabilidades das transições entre estados.

As duas condições definem uma Cadeia de Markov onde todas as probabilidades de transição entre estados podem ser também organizadas em uma estrutura algébrica denominada matriz de transição (Fig. 3). ${ }^{4}$

\footnotetext{
${ }^{4}$ Como se observa, uma matriz de transição em uma cadeia de Markov é sempre quadrada (ou seja, com números de linhas e colunas iguais). Como será discutido adiante, as dimensões da matriz de transição associada ao presente estudo não são idênticas, o que é decorrente da estratégia metodológica idealizada que possibilita o processo composicional através do software JobKov.
} 
MUSICA THEORICA Revista da Associação Brasileira de Teoria e Análise Musical 2021, v. 6, n. 1, p. 218-238 - Journal of the Brazilian Society for Music

$$
\mathrm{P}=\left[\begin{array}{cccc}
\mathrm{p}_{1,1} & \mathrm{p}_{1,2} & \ldots & \mathrm{p}_{1, \mathrm{~m}} \\
\mathrm{p}_{2,1} & \mathrm{p}_{2,2} & \ldots & \mathrm{p}_{2, \mathrm{~m}} \\
\vdots & \vdots & \vdots & \vdots \\
\mathrm{p}_{\mathrm{n}, 1} & \mathrm{p}_{\mathrm{n}, 2} & \ldots & \mathrm{p}_{\mathrm{n}, \mathrm{m}}
\end{array}\right]
$$

Figura 3: Representação genérica de uma matriz de transição com $n=m$ estados. ${ }^{5}$

A matriz de transição pode depender do tempo, ou seja, a probabilidade de transicionar de um estado i para um estado j pode depender do instante de tempo n, nesse caso, sendo a Cadeia de Markov dita, não-homogênea. Quando a matriz de transição é constante, dizemos que ela é homogênea, e esse é o caso de maior interesse. A equação (1) nos dá $\mathrm{p}_{\mathrm{i}, \mathrm{j}}$ como sendo a probabilidade de chegar a um estado futuro $X_{n+1}$ partindo de $X_{n}$.

$$
\operatorname{Pr}\left(X_{n}+1=j \mid X_{n}=i\right)=p_{i, j}
$$

onde $i, j=\{1,2, \ldots\}$

Como exemplificação em um contexto musical do que foi acima exposto, considere a melodia da canção Guten Nacht, de Brahms, transcrita, por conveniência, na tonalidade de Dó maior (Ex. 1).

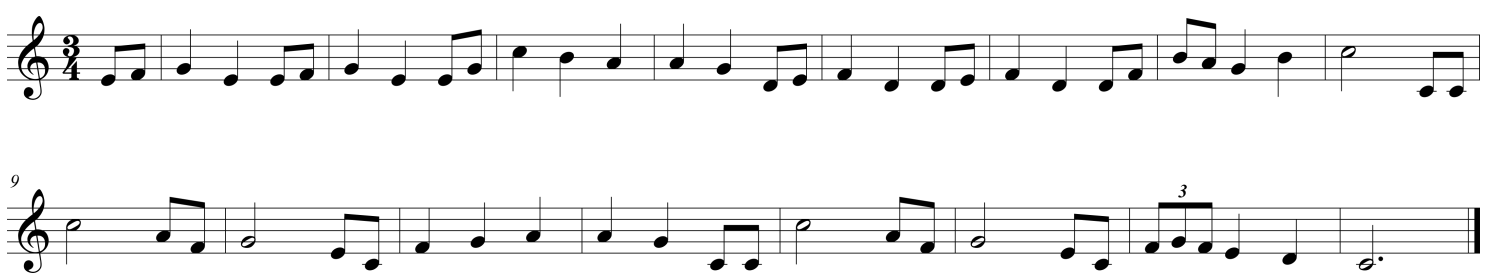

Exemplo 1: J. Brahms, Guten Nacht (c. 1-16).

Todas as sete notas diatônicas são empregadas nessa melodia, porém com frequências e "comportamentos" particulares. A Fig. 4 as dispõe como vértices

\footnotetext{
${ }^{5}$ Agradecemos ao Dr. Hugo Carvalho por gentilmente nos permitir a reprodução dos esquemas apresentados nas Figs. 1, 2 e 3 (a partir de uma palestra proferida em 2019 na Escola de Música UFRJ sobre cadeias de Markov e música), bem como pelo suporte teórico dado referente ao presente tópico.
} 
de um grafo, no qual as arestas representam conexões contíguas (ou transições, considerando a terminologia markoviana).

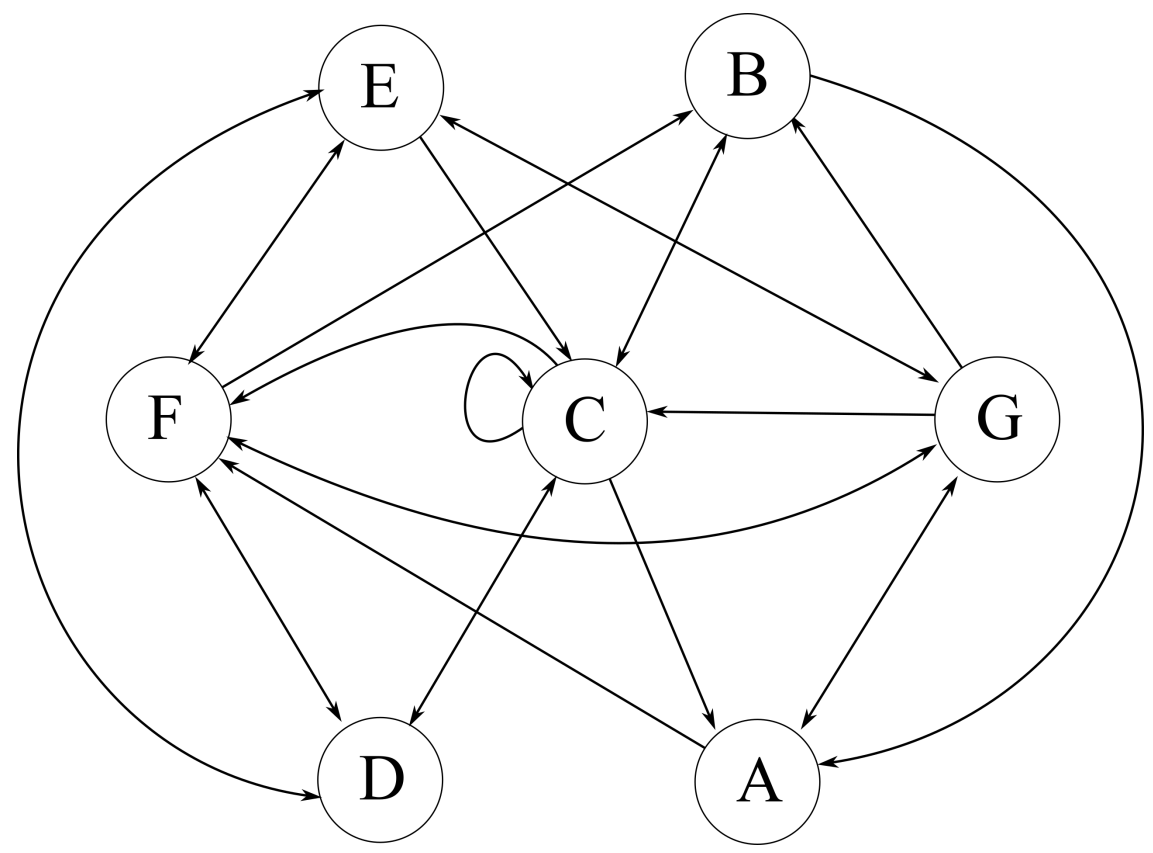

Figura 4: Transições entre as notas da melodia de Guten Nacht.

Para estabelecer o padrão melódico que caracteriza Guten Nacht, todos os caminhos (ou transições) realizados precisam ser mapeados. Isto é efetivado pela matriz de contagem ${ }^{6}$ da Fig. 5. Cada célula informa a quantidade de movimentos melódicos a partir de uma dada nota (apresentadas nas linhas da matriz) para a seguinte (dispostas nas colunas). Com a quantificação dessas transições, será posteriormente possível calcular a probabilidade de cada estado evoluir para um outro, contando o número de vezes que o respectivo caminho foi percorrido.

Subjacentemente, observa-se que, como a melodia é inteiramente diatônica, transições que envolvem notas que não pertencem à escala (sombreadas na matriz de contagem) são inexistentes (o que é evidenciado pelas linhas e colunas correspondentes, inteiramente zeradas). Visando a uma representação mais concisa e a uma eventual aplicação composicional de menor custo computacional, é possível eliminar tais linhas e colunas, obtendo-se uma versão mais compacta, denominada matriz cheia, como sugere a primeira transformação indicada na Fig. 6. Por fim, as quantidades absolutas referentes às

\footnotetext{
${ }^{6} \mathrm{Ou}$ seja, que apresenta as transições de estados de um referido contexto estudado em valores absolutos.
} 
MUSICA THEORICA Revista da Associação Brasileira de Teoria e Análise Musical 2021, v. 6, n. 1, p. 218-238 - Journal of the Brazilian Society for Music

transições são convertidas em probabilidades de ocorrência (entre 0 e 1), resultando no formato definitivo, denominado matriz de transição probabilística (segunda transformação da Fig. 6).

\begin{tabular}{|c|c|c|c|c|c|c|c|c|c|c|c|c|}
\hline & C & $C=$ & D & E, & $\mathrm{E}$ & $\mathrm{F}$ & $\mathrm{F} \#$ & G & A, & A & B, & B \\
\hline $\mathrm{C}$ & 5 & 0 & 0 & 0 & 0 & 2 & 0 & 0 & 0 & 1 & 0 & 1 \\
\hline$C \#$ & 0 & 0 & 0 & 0 & 0 & 0 & 0 & 0 & 0 & 0 & 0 & 0 \\
\hline $\mathrm{D}$ & 1 & 0 & 1 & 0 & 1 & 1 & 0 & 0 & 0 & 0 & 0 & 0 \\
\hline E, & 0 & 0 & 0 & 0 & 0 & 0 & 0 & 0 & 0 & 0 & 0 & 0 \\
\hline E & 2 & 0 & 1 & 0 & 2 & 4 & 0 & 0 & 0 & 0 & 0 & 0 \\
\hline $\mathrm{F}$ & 0 & 0 & 1 & 0 & 1 & 0 & 0 & 6 & 0 & 0 & 0 & 1 \\
\hline $\mathrm{F} \#$ & 0 & 0 & 0 & 0 & 0 & 0 & 0 & 0 & 0 & 0 & 0 & 0 \\
\hline $\mathrm{G}$ & 2 & 0 & 1 & 0 & 4 & 1 & 0 & 0 & 0 & 1 & 0 & 1 \\
\hline A, & 0 & 0 & 0 & 0 & 0 & 0 & 0 & 0 & 0 & 0 & 0 & 0 \\
\hline A & 0 & 0 & 0 & 0 & 0 & 2 & 0 & 3 & 0 & 1 & 0 & 0 \\
\hline B, & 0 & 0 & 0 & 0 & 0 & 0 & 0 & 0 & 0 & 0 & 0 & 0 \\
\hline B & 1 & 0 & 0 & 0 & 0 & 0 & 0 & 0 & 0 & 2 & 0 & 0 \\
\hline
\end{tabular}

Figura 5: Matriz de contagem referente à melodia de Guten Nacht (quantidades absolutas).
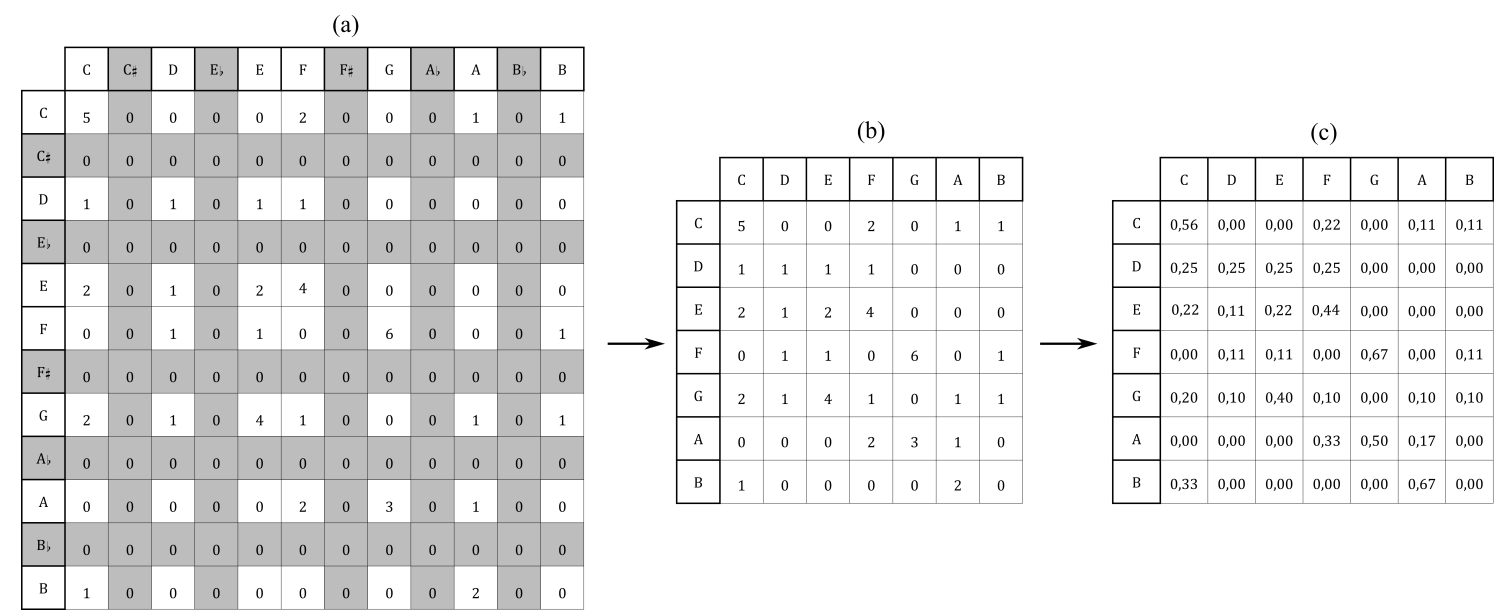

Figura 6: Conversão da matriz de contagem da Fig. 5 em matriz cheia e, em seguida, probabilística.

Ao analisar a matriz probabilística cheia é possível verificar que algumas transições se destacam mais do que outras. Nela, a probabilidade calculada de a nota Fá ser seguida pela nota Sol, por exemplo, é de 0,67 (ou 67\%). Por outro lado, algumas transições apresentam probabilidade nula, como $\mathrm{Mi} \rightarrow$ Lá, $\mathrm{Si} \rightarrow$ Ré ou Sol $\rightarrow$ Sol. Musicalmente, é possível explorar essa metodologia para investigar padrões de composição, o que se torna mais acurado quando a análise é alimentada por corpora relativamente extensos de peças. No caso do presente 
trabalho, o mapeamento de transições envolve, em vez de notas melódicas, relações harmônicas acordais presentes no cancioneiro de Jobim.

Antes de continuar com a descrição do processo composicional, convém apresentar os fundamentos que embasaram a pesquisa analítica referentes ao Sistema J.

\section{O Sistema J}

A estrutura desse sistema é baseada em conceitos e procedimentos originais, dentre os quais se destaca a noção de qualidade acordal. Entende-se aqui o termo "qualidade" como uma categoria abstrata que representa uma estrutura de acorde (considerando sua configuração estrutural e/ou as notas-funções que o compõem), independentemente de uma fundamental fixa. Ou seja, nesse sentido, uma qualidade é uma abstração de um acorde concreto. Assim, por

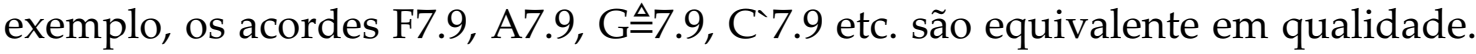
Neste artigo, eventualmente, os termos "tipo acordal" e "qualidade" serão tratados como sinônimos, já que neste contexto apenas as configurações abstratas estão em estudo.

Diretamente derivado dessa ideia está o conceito de qualidades-classes. Uma qualidade-classe (doravante identificada como Qc) é definida como um conjunto abstrato formado por tipos acordais que compartilham uma mesma base estrutural caracterizante. Contudo, os membros do conjunto diferem em termos mais superficiais, refletindo configurações particulares de tensões e alterações eventuais de suas notas-funções. Por exemplo, os tipos C7, C4.7, C7( $\triangleq 9)$, C7.9.13 representam sonoridades distintas, no entanto aparentadas pela mesma raiz acordal "dominante com sétima". Tais qualidades, portanto, integram o mesmo conjunto. Cada qualidade-classe possui um elemento gerador básico, a partir do qual todos os membros são derivados por intermédio de operações de transformação, a saber, substituição, adição e alteração cromática. Desse modo, por exemplo, o acorde C4.7 é considerado como uma variante do tipo gerador básico $\mathrm{C} 7$, pela substituição de sua terça (Mi) pela quarta (Fá). ${ }^{7}$

\footnotetext{
${ }^{7}$ Descrições detalhadas relacionadas aos diversos aspectos envolvidos na teoria das qualidadesclasses, bom como das convenções adotadas para cifragem alfanumérica, estão presentes em um livro redigido por Carlos Almada, recentemente concluído, sobre a harmonia de Jobim, atualmente em avaliação editorial.
} 
MUSICA THEORICA Revista da Associação Brasileira de Teoria e Análise Musical 2021, v. 6, n. 1, p. 218-238 - Journal of the Brazilian Society for Music

São consideradas dez qualidades-classes, como mostra o Quadro 2. As Qcs são rotuladas com as letras finais do alfabeto em ordem reversa $(Z, Y, X$, etc.). Iniciais maiúsculas representam acordes com raízes maiores (3M acima da fundamental), enquanto as minúsculas representam acordes com raízes menores (3m). Define-se ainda a forma prima de uma qualidade como uma abstração que representa todas as suas possíveis transposições. Por convenção uma forma prima tem sempre fundamental “Dó" (classe de altura “0”).

\begin{tabular}{|c|c|c|c|}
\hline \multirow{2}{*}{ Qc } & Designação informal & \multicolumn{2}{|c|}{ Forma prima geradora } \\
\cline { 3 - 4 } & & conjunto & Cifra \\
\hline Z & Maior com sétima maior & $\{0,4,7,11\}$ & $\mathrm{CM} 7$ \\
\hline Y & Maior com sétima & $\{0,4,7,10\}$ & $\mathrm{C} 7$ \\
\hline $\mathrm{X}$ & Maior com sétima e quinta diminuta & $\{0,4,6,10\}$ & $\mathrm{C}(\mathrm{b} 5) 7$ \\
\hline W & Maior com sétima e quinta aumentada & $\{0,4,8,10\}$ & $\mathrm{C}(\# 5) 7$ \\
\hline V & Tríade maior & $\{0,4,7\}$ & $\mathrm{C}$ \\
\hline z & Menor com sétima & $\{0,3,7,10\}$ & $\mathrm{Cm} 7$ \\
\hline y & "Meio diminuto" & $\{0,3,6,10\}$ & $\mathrm{C}^{\varnothing}$ \\
\hline x & Sétima diminuta & $\{0,3,6,9\}$ & $\mathrm{C}^{\circ} 7$ \\
\hline w & Menor com sétima maior & $\{0,3,7,11\}$ & $\mathrm{Cm}(\mathrm{M} 7)$ \\
\hline V & Tríade menor & $\{0,3,7\}$ & $\mathrm{Cm}$ \\
\hline
\end{tabular}

Quadro 2: Qualidades-classes acordais do Sistema-J.

A análise harmônica identificou 94 qualidades distintas nas canções de Jobim, ou seja, variantes das básicas (incluindo estas próprias), como tétrades com tensões, dominantes com quarta, ou tríades diminutas e aumentadas. Como ilustração, os quatro primeiros membros de cada Qc são apresentados no Quadro 3 em suas formas primas.

\begin{tabular}{|c|c|c|c|c|c|c|c|c|c|c|}
\cline { 2 - 11 } \multicolumn{1}{c|}{} & \multicolumn{9}{c|}{ Qcs com terça maior } & \multicolumn{5}{c|}{ Qcs com terça menor } \\
\hline $\mathrm{N}^{\circ}$ & $\mathrm{Z}$ & $\mathrm{Y}$ & $\mathrm{X}$ & $\mathrm{W}$ & $\mathrm{V}$ & $\mathrm{z}$ & $\mathrm{y}$ & $\mathrm{x}$ & $\mathrm{w}$ & $\mathrm{v}$ \\
\hline 0 & $\mathrm{CM} 7$ & $\mathrm{C} 7$ & $\mathrm{C}(65) 7$ & $\mathrm{C}(\# 5) 7$ & $\mathrm{C}$ & $\mathrm{Cm} 7$ & $\mathrm{C}^{\varnothing}$ & $\mathrm{C}^{\circ} 7$ & $\mathrm{Cm}(\mathrm{M} 7)$ & $\mathrm{Cm}$ \\
\hline 1 & $\mathrm{C} 6$ & $\mathrm{C} 4.7$ & $\mathrm{C} 4((55) 7$ & $\mathrm{C} 4(\# 5) 7$ & $\mathrm{C} 4$ & $\mathrm{Cm} 6$ & $\mathrm{C}^{\varnothing} .9$ & $\mathrm{C}^{\circ} 7.9$ & $\mathrm{Cm}(\mathrm{M} 7) 9$ & $\mathrm{Cm} .9$ \\
\hline 2 & $\mathrm{CM} 7.9$ & $\mathrm{C} 7.9$ & $\mathrm{C}(55) 7.9$ & $\mathrm{C}(\# 5) 7.9$ & $\mathrm{C} 9$ & $\mathrm{Cm} 7.9$ & $\mathrm{C}^{\varnothing} .11$ & $\mathrm{C}^{\circ} 7.11$ & $\mathrm{Cm}(\mathrm{M} 7) 11$ & $\mathrm{Cm} .11$ \\
\hline 3 & $\mathrm{C} 6 . \mathrm{M} 7$ & $\mathrm{C} 7.13$ & $\mathrm{C}(55) 7.13$ & $\mathrm{C}(\# 5) 7.13$ & $\mathrm{C} \# 5)$ & $\mathrm{Cm} 7.11$ & $\mathrm{C}^{\varnothing}((113)$ & $\mathrm{C}^{\circ} 7 . \mathrm{M} 14$ & $\mathrm{Cm}(\mathrm{M} 7) 9.11$ & $\mathrm{Cm}(55)$ \\
\hline
\end{tabular}

Quadro 3: Quatro primeiros membros de cada Qc, em forma prima.

Outro conceito de essencial importância para o presente trabalho é o de relação binária, que é definido como o tipo de conexão entre dois acordes contíguos, considerando tanto como interagem as duas qualidades quanto a distância intervalar entre suas fundamentais. 
Uma relação binária é notada simbolicamente como string, que informa concisamente as qualidades dos dois acordes relacionados (através das respectivas simbologias Qcs) e o intervalo que separa suas fundamentais (em números de semitons, considerando a possibilidade mais compacta possível). A Fig. 7 ilustra esse tipo de notação, apresentando um exemplo (a), seguido de sua generalização (b).

(a)

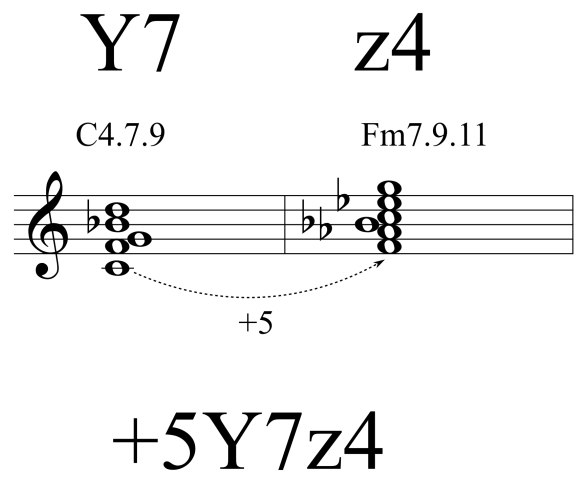

(b)

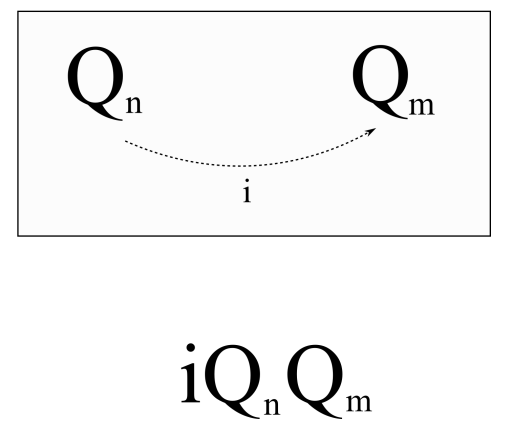

Figura 7: Notação de uma relação binária: (a) exemplo; (b) notação genérica.

$\mathrm{O}$ número de possíveis relações binárias no repertório estudado $(\mathrm{N})$ resulta do produto do total de qualidades ao quadrado (já que são dois acordes em conexão) por 12, o número possível de relações intervalares (em outros termos, classes intervalares) entre duas fundamentais quaisquer. Assim,

$$
\mathrm{N}=94^{2} \times 12=106.302
$$

Baseado nesse cálculo, foi possível, numa etapa preliminar, construir uma matriz referente às relações binárias presentes no corpus, tendo como definição 94 linhas que representam as qualidades " $\mathrm{Q}_{\mathrm{n}}$ " (ou seja, primeiro acorde da relação) e 1128 colunas que correspondem ao produto das 12 classes intervalares “i” pelas 94 qualidades " $\mathrm{Qm}$ " (o segundo acorde). A Fig. 8a apresenta a estrutura genérica dessa matriz, utilizando os rótulos das qualidades-classes do Sistema J, devidamente traduzidas para cifragens alfanuméricas em formas primas na Fig. $8 b$.

Uma questão importante surge justamente com a criação dessa matriz. Por definição, uma matriz de transição probabilística destinada a processos estocásticos markovianos deve ser quadrada, ou seja, ter exatamente o mesmo número de linhas e colunas. Ora, a matriz acima apresentada é assimétrica, com 
MUSICA THEORICA Revista da Associação Brasileira de Teoria e Análise Musical 2021, v. 6, n. 1, p. 218-238 - Journal of the Brazilian Society for Music

um número de colunas 12 vezes maior do que o de linhas. Embora essa matriz apresente propriedades estocásticas markovianas, a saber, relaciona probabilisticamente um determinado estado a outro no imediato futuro, sua estrutura não pode em stricto senso ser considerada como uma matriz de transição de uma cadeia de Markov. Na verdade, trata-se de uma variante estrutural, que será a partir deste ponto denominada matriz de transição aumentada (MTA). ${ }^{8}$ A seção seguinte descreve como o processo composicional a partir dessa solução foi devidamente idealizado, resultando na elaboração do software JobKov.

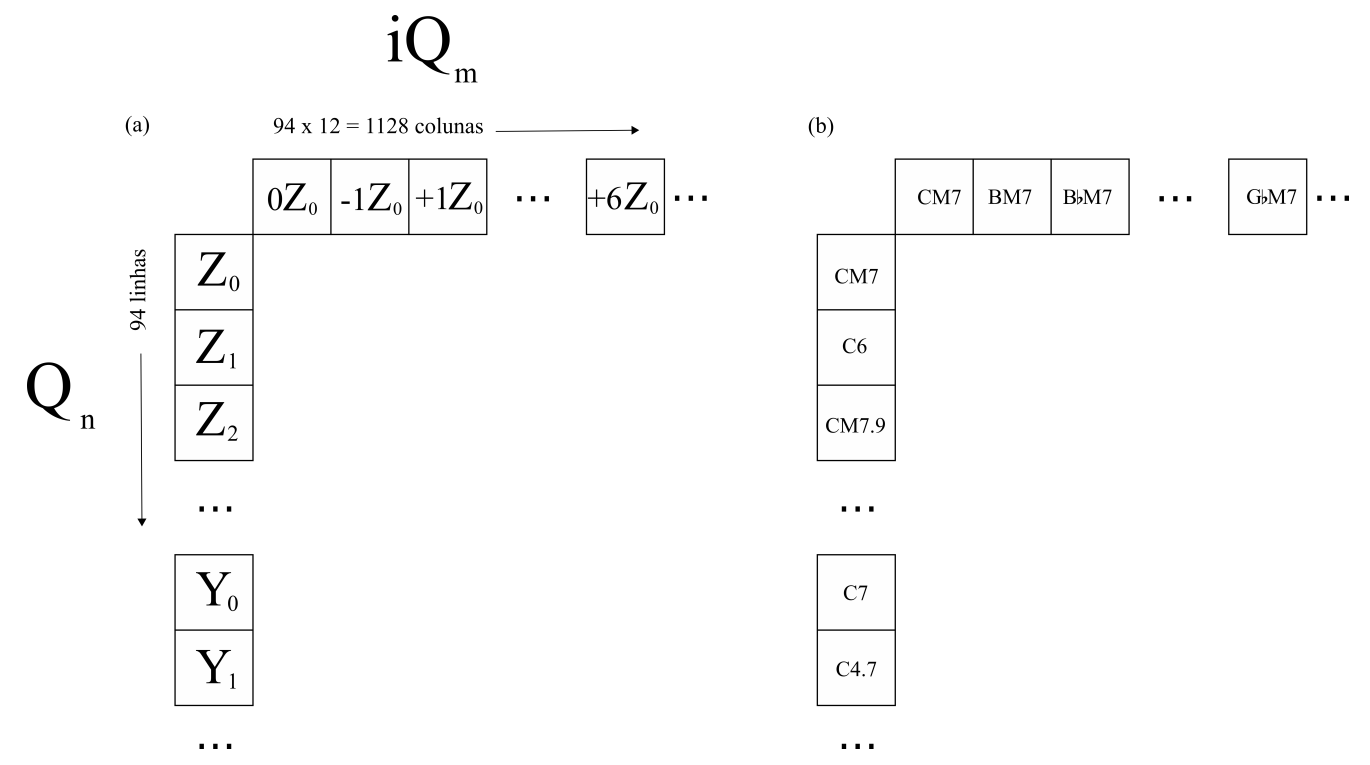

Figura 8: Estrutura básica da matriz de transição referente ao Cancioneiro Jobim em visualização parcial: a) Em formato genérico, utilizando convenções do Sistema J. b) Dispondo cifras de acordes em forma prima.

\section{O processo composicional}

A preparação do processo de composição de harmonias originais em estilo jobiniano teve seu início com a construção de MTAs para cada uma das cinco fases jobinianas, a partir dos dados obtidos pela análise. A ideia por trás dessa estratégia seria propiciar ao usuário do futuro programa a obtenção de harmonias que refletissem particularidades estilísticas do compositor, no que se

\footnotetext{
${ }^{8}$ Agradecemos novamente ao Dr. Hugo Carvalho pelas informações sobre essa peculiaridade matricial e pelo nome sugerido.
} 
refere ao aspecto harmônico, em diferentes momentos de sua carreira. De modo semelhante, uma MTA abrangendo o corpus completo foi também construída.

Ainda que haja pequenas diferenças em relação às dimensões das seis matrizes (já que as fases variam em número de acordes e relações binárias empregados), de um modo geral, seus gigantescos tamanhos revelaram-se como um sério obstáculo para a viabilização do processo de composição algorítmica. A manipulação de dados em estruturas de tal magnitude causaria, na melhor das hipóteses, alto custo computacional e demandaria muito tempo de processamento.

A solução para o problema foi idealizada a partir da ideia de transformar cada MTA em versões "cheias" (como exemplificado com a melodia de Brahms no início deste artigo), formando estruturas matriciais denotadas, a partir deste ponto, como MTA*. Tal estratégia permitiu uma redução dramática das dimensões das matrizes, tendo em vista que dentro do conjunto de alternativas possíveis de transições de um determinado estado A (acordes nas linhas de uma MTA) para um estado B (intervalos + acordes nas colunas), Jobim, de acordo com o que foi apurado nas análises, escolheu, em média, menos do que 5\% do total. Esse índice restrito é extremamente significativo, pois nos leva a considerar tal subconjunto de escolhas como representativo do estilo harmônico do compositor. ${ }^{9}$ O Quadro 4 expõe os números referentes a tais dados em todas as cinco fases e no corpus completo.

\begin{tabular}{|c|c|c|c|c|c|c|c|}
\cline { 2 - 8 } \multicolumn{1}{c|}{} & \multicolumn{2}{c|}{ MTA* $^{*}$} & \multicolumn{4}{c|}{ Relações binárias } \\
\hline Fase & Linhas & Colunas & Possíveis & "Não-jobinianas" & \multicolumn{2}{c|}{ Jobinianas } \\
\hline 1 & 66 & 302 & 19.932 & 18.982 & $95.2 \%$ & 951 & $4.8 \%$ \\
\hline 2 & 79 & 394 & 31.126 & 29.831 & $95.8 \%$ & 1.295 & $4.2 \%$ \\
\hline 3 & 49 & 156 & 7.595 & 7.355 & $96.2 \%$ & 289 & $3.8 \%$ \\
\hline 4 & 63 & 270 & 17.010 & 16.314 & $95.9 \%$ & 696 & $4.1 \%$ \\
\hline todas & 94 & 547 & 51.418 & 48.723 & $94.8 \%$ & 2.695 & $5.2 \%$ \\
\hline
\end{tabular}

Quadro 4: Dimensões das MTAs cheias (MTA*) e relações binárias envolvidas, destacando o subconjunto de escolhas jobinianas (em valores absolutos e percentuais).

Formadas as respectivas MTA*s, o processo composicional se desenrolou de maneira relativamente simples, podendo ser descrito pelo seguinte algoritmo:

\footnotetext{
${ }^{9}$ Essa afirmação é suportada pela linha de pesquisa que considera o aprendizado estatístico (statistical learning) como um fator decisivo para o mapeamento de um estilo. Tal tese, inaugurada por Leonard Meyer (1956), é atualmente desenvolvida teórica e metodologicamente por pesquisadores como David Huron (2006) e David Temperley (2007), entre outros.
} 
1. Determinação do acorde $\mathbf{A}^{10}$ (qualidade $Q_{\mathrm{n}}$ ) - ou seja, escolha do acorde que dará início ao processo e que encabeçará a progressão harmônica a ser composta. A seleção pode ser feita de duas maneiras (ambas devidamente implementadas no software): aleatória (através de sorteio equiprovável entre as alternativas das linhas da MTA* em questão) ou determinística, pelo próprio usuário (também dentre as opções que integram as linhas);

2. Criação do vetor de probabilidades - o programa localiza a linha da $\mathrm{MTA}^{*}$ correspondente ao acorde do estado atual (o acorde A, neste caso), produzindo uma cópia que se transforma no vetor de probabilidades vp referente ao acorde em questão, cujos elementos são suas possíveis transições;

3. Sorteio da transição - uma função específica realiza um sorteio markoviano, i.e., estabelecendo pesos distintos proporcionais às magnitudes dos elementos do vetor vp. Desse modo, maiores probabilidades (que representam em última instância escolhas mais recorrentes de Jobim) têm maior chance de serem selecionadas; ${ }^{11}$

4. Identificação do acorde B - como a coordenada da probabilidade sorteada corresponde à coluna da $\mathrm{MTA}^{*}$, a identificação do acorde de transição é assim diretamente obtida;

5. Conversão de $\mathbf{B}$ em $\mathbf{Q}_{\mathbf{n}}$ - um novo ciclo da progressão harmônica se inicia com a conversão do acorde de transição B em qualidade $Q_{n}$. Como as linhas da MTA* (onde habitam os acordes $Q_{n}$ ) contêm apenas qualidades em forma prima, o programa deve primeiro normalizar o acorde B (ou seja, transpor sua fundamental para "Dó") para, em seguida, buscar a linha correspondente à nova qualidade na estrutura matricial. A partir desse ponto, o processo replica as etapas 2 a 5, até que a extensão (em números de acordes) desejada pelo usuário do programa seja alcançada.

A Fig. 9 resume as etapas do algoritmo em uma representação gráfica, tomando como exemplo de semente a qualidade $\mathrm{Cm} 7$.

\footnotetext{
10 Informalmente também designado como "acorde-semente".

${ }^{11}$ É importante enfatizar que os pesos refletem tendências e não certezas de escolhas, o que dá ao processo uma pequena, mas existente margem de imponderabilidade, essencial para que as harmonias compostas não sejam puramente reproduções de escolhas jobinianas.
} 


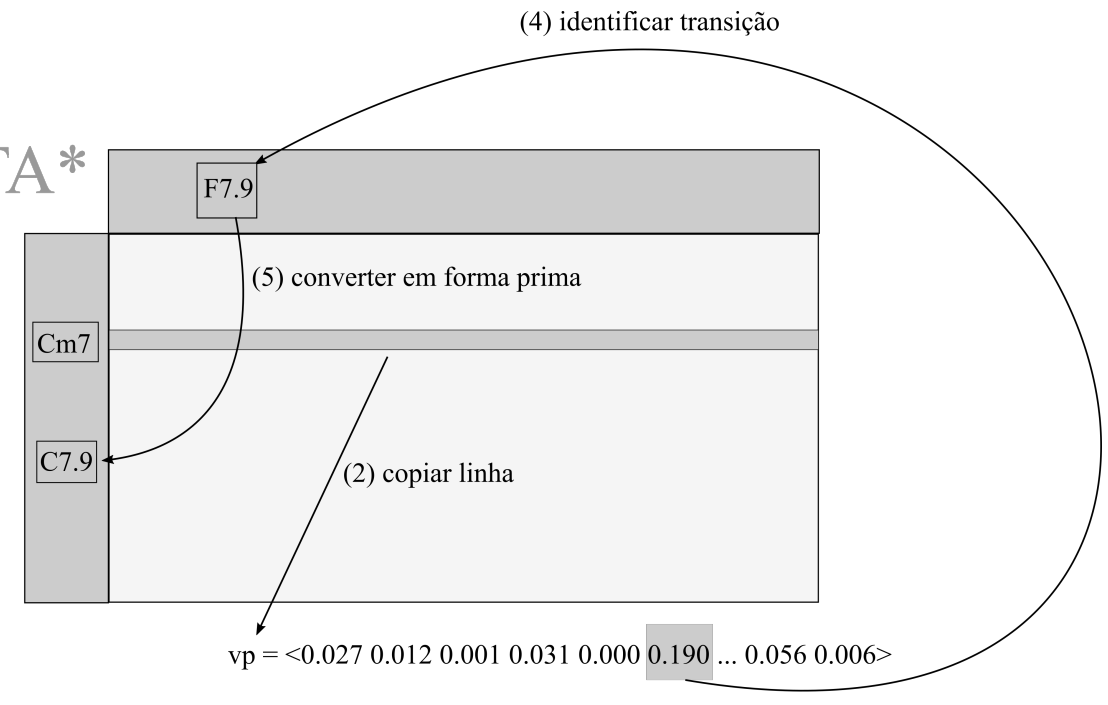

(3) sortear transição

Replicar etapas 2-5 até o final da progressão

Figura 9: Representação gráfica do algoritmo básico de composição por JobKov, tomando como exemplo a qualidade $\mathrm{Cm} 7$ como acorde-semente.

\section{Descrição do programa JobKov}

JobKov foi desenvolvido para processar os dados obtidos e criar progressões de acordes originais, estatisticamente condizentes com as escolhas de Jobim. Esse processamento é feito através de escolhas estocásticas condicionadas pela maior ou menor recorrência de relações binárias com base nos dados obtidos das análises, retornando progressões com extensões determinadas pelo usuário. Uma vez que cada uma das fases composicionais de Jobim apresenta distintas preferências no que tange às relações binárias acordais, o programa possibilita que o usuário selecione entre as cinco fases criativas (ou considerando todo o corpus) qual baseará a progressão a ser criada.

A interface do programa, apresentada na Fig. 10, é de simples operação por um usuário, que inicialmente seleciona a fase jobiniana desejada para sua progressão (A). Em seguida, determina o acorde-semente, digitando a qualidade-classe escolhida no campo (B), ou deixando que a escolha seja feita aleatoriamente pelo programa, acionando o botão "RANDOM" (C). Ao pressionar o botão "COMPOR" (E), o programa cria uma progressão harmônica, seja com a extensão padronizada (quatro relações binárias ou cinco acordes no total) ou com qualquer outro número digitado no campo (D). A progressão é 
MUSICA THEORICA Revista da Associação Brasileira de Teoria e Análise Musical 2021, v. 6, n. 1, p. 218-238 - Journal of the Brazilian Society for Music

então publicada no campo $(\mathrm{F})$, como sequência de cifras alfanuméricas (por default, iniciando-se com a forma prima do acorde-semente). A progressão criada pode então ser salva como arquivos em formatos ttxt e .mid (G). O botão "PLAY" (H) permite que o usuário ouça a progressão quantas vezes desejar.

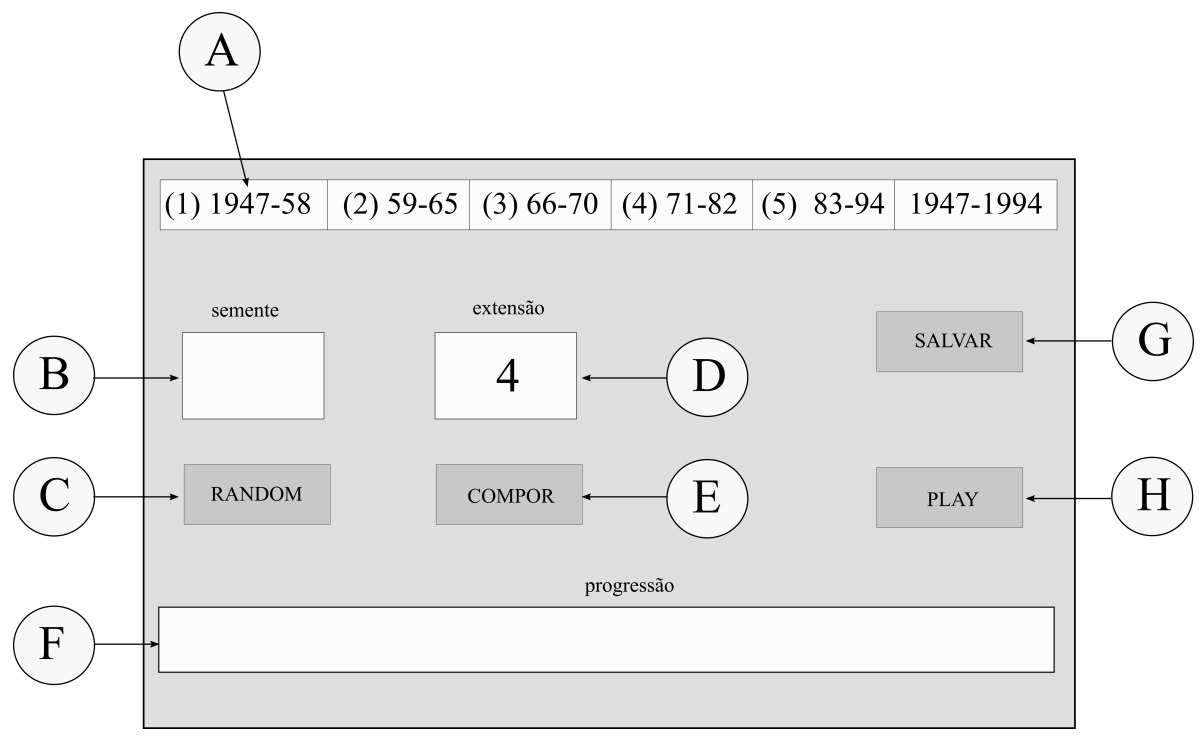

Figura 10: Interface gráfica de Jobkov.

O Quadro 5 apresenta seis progressões compostas por JobKov em modo aleatório, cada qual contemplando uma das fases. ${ }^{12}$

\begin{tabular}{|c|c|c|c|c|c|c|}
\cline { 2 - 7 } \multicolumn{1}{c|}{} & \multicolumn{5}{c|}{ acordes } \\
\hline fase & 1 & 2 & 3 & 4 & 5 & 6 \\
\hline 1 & $\mathrm{C} 7.9(b 13)$ & $\mathrm{Fm} 7.9$ & $\mathrm{~B} b 7.9$ & $\mathrm{G} 7.9 .13$ & $\mathrm{E} b 7.9(\# 11)$ & $\mathrm{A} b \mathrm{M} 7$ \\
\hline 2 & $\mathrm{CM} 7.9$ & $\mathrm{Cm}(\mathrm{M} 7) 9.11$ & $\mathrm{Bm} 7$ & $\mathrm{E} 7$ & $\mathrm{E} b 7$ & $\mathrm{~A} b(\# 5) 7(b 9)$ \\
\hline 3 & $\mathrm{C} 4.7(b 9 . b 13)$ & $\mathrm{Fm} .9$ & $\mathrm{Fm} 7$ & $\mathrm{Fm} 6$ & $\mathrm{Fm} 6.9$ & $\mathrm{G} 4(b 5) 7(b 9)$ \\
\hline 4 & $\mathrm{CM} 7.9(\# 11)$ & $\mathrm{F} 7$ & $\mathrm{~B} b(\# 5) 7$ & $\mathrm{D}^{\circ} 7$ & $\mathrm{~B} b \mathrm{~m}$ & $\mathrm{C} 7$ \\
\hline 5 & $\mathrm{C} 7(b 13)$ & $\mathrm{F} 7.9$ & $\mathrm{~B} b 7(b 9) 13$ & $\mathrm{E} b 6.9$ & $\mathrm{Cm} 7$ & $\mathrm{~F} 7.9 .13$ \\
\hline todas & $\mathrm{C}^{\circ} 7(b 13)$ & $\mathrm{Bm} 7$ & $\mathrm{CM} 7$ & $\mathrm{FM} 7(\# 11)$ & $\mathrm{F} 7(b 9 . \# 11)$ & $\mathrm{B} b \mathrm{M} 7$ \\
\hline
\end{tabular}

Quadro 5: Exemplos de progressões harmônicas com seis acordes criadas por JobKov.

\section{Considerações finais e trabalhos futuros}

Este artigo teve como principal objetivo a descrição dos processos estocásticos/markovianos idealizados para a criação de harmonias originais e

\footnotetext{
12 Importante acrescentar que esses exemplos foram produzidos a partir de seis aplicações sequenciais do programa, sem quaisquer edição ou seleção.
} 
estilisticamente associadas à prática de Jobim. Tais processos resultaram na elaboração do software JobKov, que possui grande potencial para aplicações composicionais e pedagógicas. Pesquisas correlatas, envolvendo repertórios de outros compositores foram já iniciadas, utilizando o mesmo aparato computacional. É o caso de composições de Bill Evans e Ivan Lins (já concluídas) e de Chico Buarque de Holanda e Edu Lobo (em andamento). De maneira similar ao que foi feito com o corpus Jobim, a mesma tecnologia com MTAs* e softwares de composição mostrou-se perfeitamente aplicável, com resultados igualmente satisfatórios.

Subjacentemente, o estudo introduziu e discutiu, ainda que brevemente, um elemento de grande importância para a pesquisa na qual se insere, a saber, o fator de aprendizado estatístico na determinação de um estilo. Nesse sentido, parece-nos extremamente significativo o fato de que as escolhas de acordes e seus encadeamentos (relações binárias nos presentes termos) forme um pequeno subconjunto do total de possibilidades.

É justamente essa reflexão que desponta como um dos mais promissores caminhos para exploração da pesquisa. A combinação das ideias de expectativa, entropia, estilo com levantamento estatístico dos dados da análise harmônica aqui descrita forma o cerne de um estudo teórico atualmente em preparação, visando contribuir para a sistemática expansão do conhecimento sobre a extraordinária obra musical de Jobim..$^{13}$

\section{Referências}

1. Almada, Carlos. A harmonia de Jobim (livro em processo de avaliação editorial).

2. Almada, Carlos; Kühn, Max, Usai, Claudia, Chagas, Igor, Penchel, João. 2020. A geometria das relações parcimoniosas em música popular. In: Anais do Congresso Nacional de Música e Matemática, 2, 2017. Rio de Janeiro: UFRJ, p. 1428.

3. Almada, Carlos; Braga, Vinícius; Cabral, Eduardo; Chagas, Igor; Kühn, Max; Miccolis, Ana; Usai, Claudia. 2019a. J-Analyzer: A Software for Computer-

\footnotetext{
${ }^{13}$ Gostaríamos de expressar nossa sincera gratidão aos avaliadores anônimos deste artigo pela pertinência de seus comentários e sugestões, o que contribuiu para o aperfeiçoamento do estudo.
} 
Assisted Analysis of Antônio Carlos Jobim's Songs. In: Proceedings of Symposium of the Brazilian Society of Computer Music, 17. São João del Rei: UFSJ.

4. Almada, Carlos; Braga, Vinícius; Cabral, Eduardo; Chagas, Igor; Kühn, Max; Miccolis, Ana; Usai, Claudia. 2019b. An Analytical Methodology for Harmonic Analysis of Antônio Carlos Jobim's Songs. In: Symposium of Music Theory and Analysis (Música Analítica). Porto: Centro de Investigação em Ciência e Tecnologia das Artes.

5. Cabral, S. Antonio Carlos Jobim: uma biografia. Rio de Janeiro: Lumiar, 1997.

6. Carvalho, Hugo. 2019. An Introduction to Markov Chains in Music Composition and Analysis. MusMat: Brazilian Journal of Music and Mathematics, v. 3, n. 2, p. 18-43.

7. Castro, Ruy. A noite de meu bem: a história e as histórias do samba-canção. São Paulo: Companhia das Letras, 2015.

8. Castro, Ruy. A onda que se ergueu no mar: novos mergulhos na bossa-nova. São Paulo: Companhia das Letras, 2001.

9. Castro, Ruy. Chega de saudade. São Paulo: Companhia das Letras, 1990.

10. Huron, David. 1992. Sweet Anticipation: Music and the Psychology of Expectation. Cambridge: The MIT Press.

11. Jobim, Antonio Carlos. 2006. Cancioneiro Jobim: Obras Completas (5 vol.). Rio de Janeiro: Jobim Music.

12. Jobim, Helena. Antonio Carlos Jobim: um homem iluminado. Rio de Janeiro: Nova Fronteira, 1996.

13. Kühn, Max, Usai, Claudia, Chagas, Igor, Penchel, João; Almada, Carlos. 2017. Relações neorriemanianas de acordes de sétima na primeira fase composicional de Antônio Carlos Jobim. In: Anais do Encontro Internacional de Teoria e Análise Musical, 4, 2017. São Paulo: USP, p. 174-183.

14. Lewin, David. 1987. Generalized Musical Intervals and Transformations. New Haven: Yale University Press.

15. Meyer, Leonard. 1956. Emotion and Meaning in Music. Chicago: University of Chicago Press.

16. Moss, Fabian et al. 2019. Statistical Characteristics of Tonal Harmony: A Corpus Study of Beethoven's String Quartets. Plos One. Disponível em: <https://doi.org/10.1371/journal.pone.0217242>. Acesso em 15/out./2020.

17. Rings, Steve. 2011. Tonality and Transformation. Oxford: Oxford University Press. 
18. Temperley, David; Clrecq, Trevor de. 2013. Statistical Analysis of Harmony and Melody in Rock Music. Journal of New Music Research, v. 42, n. 3, p. 187204.

19. Temperley, David. 2007. Music and Probability. Cambridge: The MIT Press.

20. Usai, Claudia; Kühn, Max; Chagas, Igor; Penchel, João. 2020. Relações entre acordes de sétima no cancioneiro de Antônio Carlos Jobim. In: Anais do Colóquio de Pesquisa do Programa de Pós-Graduação Em Música da UFRJ, 17, 2018. Rio de Janeiro: UFRJ. 